\title{
Extreme topological measures
}

\author{
by \\ S. V. Butler (Urbana-Champaign, IL)
}

\begin{abstract}
It has been an open question since 1997 whether, and under what assumptions on the underlying space, extreme topological measures are dense in the set of all topological measures on the space. The present paper answers this question. The main result implies that extreme topological measures are dense on a variety of spaces, including spheres, balls and projective planes.
\end{abstract}

1. Introduction. A topological measure on a compact Hausdorff space is a real-valued, nonnegative, monotone, countably additive and regular set function defined on the family of sets that are either closed or open. Topological measures on $X$ correspond to functionals that are linear on singly generated subalgebras of $C(X)$ and, hence, generalize linear functionals on $C(X)$. See [2] for details. The fact that a topological measure has all the properties of a regular Borel measure, except that it is only defined on closed and open sets, has a profound effect on characteristics of topological measures compared with those of regular Borel measures.

Here we consider the nature of extreme topological measures, i.e. extreme points of the space of all topological measures on a given set. It is known (see [7]) that the collection of extreme topological measures has a rich structure and includes different types of topological measures that are not $\{0,1\}$-valued. It has been an open question since 1997 whether, and under what assumptions on the underlying space, extreme topological measures are dense in the set of all topological measures on the space. In the present paper we answer this question.

If $E$ is a set, we will denote by $|E|$ its cardinality and by $\mathcal{P}(E)$ its power set. In this article we will often use disjoint collections of sets. This means that if $\mathcal{D}$ is a disjoint collection and $U, V \in \mathcal{D}$ then either $U=V$ or $U \cap V=\emptyset$. We use the symbol $\bigsqcup$ for the union of sets that belong to a

2000 Mathematics Subject Classification: 46E27, 28C15, 54F65.

Key words and phrases: extreme topological measures, supermeasures, dense subset, solid sets, solidifiable sets, solid extension, $m$-chains. 
disjoint family of sets. For example, $\bigsqcup \mathcal{H}=\bigsqcup_{A \in \mathcal{H}} A$ means that we take the union of sets in a disjoint family $\mathcal{H}$. If $\mathcal{W}$ is a finite collection of subsets of a space $X$, we will denote by $\operatorname{DC}(\mathcal{W}) \subseteq \mathcal{P}(\mathcal{W})$ the set of disjoint subfamilies of $\mathcal{W}$. We will also use $\operatorname{MDC}(\mathcal{W}) \subseteq \operatorname{DC}(\mathcal{W})$, the set of all maximal elements of $\operatorname{DC}(\mathcal{W})$ with respect to inclusion. An element $\mathcal{D} \in \operatorname{MDC}(\mathcal{W})$ is a maximal disjoint collection of sets from $\mathcal{W}$.

Definition 1.1. Let $X$ be a compact Hausdorff space. Let $\mathcal{C}(X)$ (respectively $\mathcal{O}(X))$ denote the collection of closed (respectively open) subsets of $X$, and $\mathcal{A}(X)=\mathcal{C}(X) \cup \mathcal{O}(X)$. A topological measure on $X$ is a function $\mu: \mathcal{A}(X) \rightarrow \mathbb{R}^{+}$such that:

(i) $\mu\left(\bigsqcup_{i=1}^{n} A_{i}\right)=\sum_{i=1}^{n} \mu\left(A_{i}\right)$ (all $A_{i}$ and $\bigsqcup_{i=1}^{n} A_{i}$ are assumed to be in $\mathcal{A}(X))$

(ii) $\mu(U)=\sup \{\mu(C): C \subseteq U, C \in \mathcal{C}(X)\}$ for all $U \in \mathcal{O}(X)$.

From (i) and (ii) it follows that topological measures are monotone. Topological measures are not only finitely, but also countably additive: if $A=\bigsqcup_{i=1}^{\infty} A_{i}$, where $A, A_{i} \in \mathcal{A}(X)(i=1,2, \ldots)$, then $\mu(A)=\sum_{i=1}^{\infty} \mu\left(A_{i}\right)$ (see [10]).

Topological measures on a given set $X$ form a convex set, and the extreme points of this convex set will be called extreme topological measures. Topological measures that only assume values 0 and 1 are called simple. Any simple topological measure is extreme.

Definition 1.1 involves open and closed sets. We frequently use special open or closed sets. A set $A$ is solid if both $A$ and its complement are connected. We will denote collections of open solid sets and of closed solid sets by $\mathcal{O}_{\mathrm{s}}(X)$ and $\mathcal{C}_{\mathrm{s}}(X)$ respectively. We will also use $\mathcal{A}_{\mathrm{s}}(X)=\mathcal{O}_{\mathrm{s}}(X) \cup$ $\mathcal{C}_{\mathrm{S}}(X)$. To use the technique of solid sets (Theorem 2.2 below) we assume that $X$ is connected and locally connected. We shall also assume for simplicity that $X$ has a certain topological characteristic, genus $g=0$ (see [3] for details). Intuitively, $X$ does not have holes or loops. One way to describe the " $g=0$ " condition is the following: if the union of two open solid sets in $X$ is the whole space, their intersection must be connected (see [8]). Note also that a space has genus 0 if and only if $U \backslash C$ is connected for any open connected set $U$ and any closed solid set $C$ contained in $U$. (See Remark 2.6 below.) In the case where $X$ is locally path connected, $g=0$ if the fundamental group $\pi_{1}(X)$ is finite (in particular, if $X$ is simply connected) or, more generally, if $H^{1}(X)=0([9,12])$. A compact, connected, locally connected Hausdorff space with genus 0 will be called a $q$-space. Examples of $q$-spaces include spheres $S^{n}$, balls $B^{n}$ for $n \geq 2$, and dendrites.

In this paper $X$ is always a $q$-space, and all topological measures are assumed to be normalized, i.e. $\mu(X)=1$. On the space of all normalized topological measures on $X$, denoted by $T M(X)$, we consider the weak* 
topology which is generated by basic open sets

$$
\widehat{U}(\mathcal{W}, b)=\{\mu \in T M(X): \mu(U)>b(U) \text { for all } U \in \mathcal{W}\},
$$

where $\mathcal{W}$ is a finite collection of open solid sets, and $b: \mathcal{W} \rightarrow[0,1]$ is a function. The space $T M(X)$ is a compact Hausdorff convex topological space. (See [1]; a more general result is in [11].)

\section{Preliminaries}

Definition 2.1. A solid set function on a $q$-space $X$ is a function $\mu$ : $\mathcal{A}_{\mathrm{s}}(X) \rightarrow[0,1]$ such that

1. if $C_{1} \sqcup \cdots \sqcup C_{n} \subseteq C, C, C_{1}, \ldots, C_{n} \in \mathcal{C}_{\mathrm{s}}(X)$, then $\sum_{i=1}^{n} \mu\left(C_{i}\right) \leq \mu(C)$,

2. $\mu(U)=\sup \left\{\mu(C): C \subseteq U, C \in \mathcal{C}_{\mathrm{s}}(X)\right\}$ for $U \in \mathcal{O}_{\mathrm{s}}(X)$,

3. $\mu(U)+\mu(X \backslash U)=1$ for $U \in \mathcal{O}_{\mathrm{s}}(X)$.

The proof of the next theorem is in [3].

TheOREM 2.2. A solid set function on a q-space extends uniquely to a topological measure on $X$.

Now we give two examples of topological measures that first appeared in [2] and [3]. These topological measures are not subadditive, hence they are not Borel measures.

ExAMPLE 2.3. Let $X$ be the unit square and $B$ be the boundary of $X$. Fix a point $p$ in $X \backslash B$. Define $\mu$ on solid sets as follows: $\mu(A)=1$ if (i) $B \subset A$, or (ii) $p \in A$ and $A \cap B \neq \emptyset$. Otherwise, we let $\mu(A)=0$. Then $\mu$ is a solid set function and hence extends to a topological measure on $X$. To demonstrate that $\mu$ is not a measure we shall show that $\mu$ is not subadditive. Let $A_{1}$ be a closed solid set consisting of two adjacent sides of $B$, let $A_{2}$ be a closed solid set that is the other two adjacent sides of $B$, and $A_{3}=X \backslash B$ be an open solid subset of $X$. Then $X=A_{1} \cup A_{2} \cup A_{3}, \mu(X)=1$, but $\mu\left(A_{1}\right)+\mu\left(A_{2}\right)+\mu\left(A_{3}\right)=0$.

ExAmple 2.4. Let $X$ be a sphere. Fix three points $x, y, z$ in $X$. Define $\mu$ on solid sets as follows: $\mu(A)=1$ if $A$ contains the majority of the three points, otherwise $\mu(A)=0$. The resulting topological measure is not subadditive, since $\mu(X)=1$, and it is easy to represent $X$ as a union of three overlapping solid sets each of which contains exactly one of the points $x, y, z$. Notice also that $\mu(A)=1$ for any connected set $A$ that contains at least two points. This follows easily from Lemmas 3.1 and 3.2 in [3].

Proposition 2.5. In a q-space, $U \backslash C$ is connected for any open connected set $U$ and any closed solid set $C$ contained in $U$. In particular, in a q-space with no cut points, $U \backslash\{x\}$ is connected for any open connected set $U$ and any point $x$. 
Proof. We give a proof, suggested by D. Grubb, that uses topological measures. Observe first that $C$ intersects the closure of every component of $U \backslash C$. Indeed, let $U \backslash C=\bigsqcup_{\beta} V_{\beta}$ and assume that $C \cap V_{\alpha}=\emptyset$ for some component $V_{\alpha}$. All components are open by local connectivity, so $\bar{V}_{\alpha} \cap V_{\beta}=\emptyset$ for all $\beta \neq \alpha$. Then $V^{\prime}=U \backslash \bar{V}_{\alpha}=\bigsqcup_{\beta \neq \alpha} V_{\beta} \sqcup C$ is an open set and hence $U=V_{\alpha} \sqcup V^{\prime}$ is a disconnection of $U$.

Now, if $V_{1}$ and $V_{2}$ are two components of $U \backslash C$, take $x \in C, y \in V_{1}$, $z \in V_{2}$. Let $\mu$ be the simple topological measure based on points $x, y, z$ as in Example 2.4. Note that $\mu(U)=1, \mu(C)=0$, so $\mu(U \backslash C)=1$. Pick the component $V_{\alpha}$ of $U \backslash C$ with $\mu\left(V_{\alpha}\right)=1$. Since $V_{\alpha}$ cannot contain both $y$ and $z$, we may assume that $y \notin V_{\alpha}$, i.e. $V_{\alpha} \neq V_{1}$. Then $x$ and $y$ must be in different components of $X \backslash V_{\alpha}$ because $\mu\left(X \backslash V_{\alpha}\right)=0$. But then $C$ and $V_{1}$ are contained in different components of $X \backslash V_{\alpha}$. So $C \cap \bar{V}_{1}=\emptyset$ (note that components of $X \backslash V_{\alpha}$ are closed), which contradicts the statement at the beginning of the proof.

Remark 2.6. From Propositions 2.5 and 2.2 in [3] we get a characterization of genus 0 . A compact Hausdorff connected locally connected space has genus 0 if and only if $U \backslash C$ is connected for any open connected set $U$ and any closed solid set $C$ contained in $U$.

Definition 2.7. A topological measure $\mu$ on $X$ is finitely defined if there is a finite subset $F \subset X$ such that $\sum_{i=1}^{n} \mu\left(A_{i}\right) \leq \mu(A)$ whenever $\bigsqcup_{i=1}^{n}\left(A_{i} \cap F\right) \subseteq A \cap F$, where $A, A_{1}, \ldots, A_{n} \in \mathcal{A}_{\mathrm{s}}(X)$.

The topological measure in Example 2.4 is finitely defined, but the one in Example 2.3 is not. Both topological measures are simple, hence extreme.

Definition 2.8. A supermeasure on a finite set $E$ is a function $\nu$ : $\mathcal{P}(E) \rightarrow[0,1]$ satisfying

1. $\nu(A)+\nu(E \backslash A)=1$ for all $A \in \mathcal{P}(E)$,

2. $\nu(A \sqcup B) \geq \nu(A)+\nu(B)$.

The second condition says that $\nu$ is superadditive, hence the term supermeasure. From the definition it immediately follows that $\nu(\emptyset)=0$ and that $\nu$ is monotone, i.e. $J \subseteq K \Rightarrow \nu(J) \leq \nu(K)$. From (ii) we also deduce (by induction) that if $\bigsqcup_{i} J_{i} \subseteq J$ then $\sum_{i} \nu\left(J_{i}\right) \leq \nu(J)$.

The reason behind the introduction of supermeasures is demonstrated by the next two results (see [4]).

Proposition 2.9. Let $E$ be a finite subset of $X$, and let $i: E \rightarrow X$ be the inclusion. For any supermeasure $\nu$ on $E$ let

$$
\mu(A)=i_{*} \nu(A)=\nu(A \cap E), \quad A \in \mathcal{A}_{\mathrm{s}}(X) .
$$

Then $\mu$ is a solid set function on $X$, and it uniquely extends to a topological measure $\mu=i_{*} \nu$ which is finitely defined. 
Definition 2.10. A pre-supermeasure on a finite set $E$ is a function $\alpha: \mathcal{F} \rightarrow[0,1]$, where $\mathcal{F}$ is a subset of $\mathcal{P}(E)$, such that

$$
\bigsqcup_{A \in \mathcal{H}} A \subseteq B \text { implies } \sum_{A \in \mathcal{H}} \alpha(A) \leq \alpha(B) .
$$

TheOREM 2.11. If $|E|$ is odd, $\mathcal{F} \subseteq \mathcal{P}(E)$ is a family containing $E$ and $\alpha: \mathcal{F} \rightarrow[0,1]$ is a pre-supermeasure, then there exists a supermeasure $\nu$ on $E$ such that $\nu(A) \geq \alpha(A)$ for all $A \in \mathcal{F}$ and $\nu(A)=\alpha(A)$ for all $A \in \mathcal{F}$ with $|A| \leq \frac{1}{2}|E|$.

This is Lemma 2.5 in [4] without the condition $\emptyset \notin \mathcal{F}$. (From the way $\nu$ is defined it is clear that $\nu(\emptyset)=0$, whether or not $\emptyset \in \mathcal{F}$.) The proof also shows that $\nu(A)=\alpha(A)$ for all $A \in \mathcal{F}$ with $|A| \leq \frac{1}{2}|E|$.

Definition 2.12. A family $\mathcal{F}$ of nonempty sets has order $m$ if $\mathcal{F}$ contains a collection of $m$ disjoint sets but no collection of $m+1$ disjoint sets.

Definition 2.13. Let $\mathcal{C}=\left\{I_{1}, \ldots, I_{k}\right\}$ be a sequence of distinct sets. Let $m \leq k$ be an integer. We say that $\mathcal{C}$ is an $m$-disjoint $k$-chain if $\mathcal{C}$ has order $m$ and for each $i=1, \ldots, k$ the sets $I_{i+1}, \ldots, I_{i+m}$ are disjoint (indices are $\bmod k$ ). An $m$-disjoint $(2 m+1)$-chain is called an $m$-chain.

EXAMPLE 2.14. If $m$ is an integer and $\mathcal{S}=\left\{S_{1}, \ldots, S_{2 m+1}\right\}$ is a disjoint family of sets, then $\left\{J_{i}=S_{2 i-1} \sqcup S_{2 i}: i=1, \ldots, 2 m+1(\bmod 2 m+1)\right\}$ is an $m$-chain. Such $m$-chains play an important role in this paper, and we give them a special name.

Definition 2.15. An $m$-chain $\left\{J_{1}, \ldots, J_{2 m+1}\right\}$ that arises from a disjoint family of $2 m+1$ sets as in Example 2.14 is called a basic $m$-chain.

LEMMA 2.16. If $\left\{A_{1}, \ldots, A_{k}\right\}$ is an $m$-disjoint $k$-chain of sets from $\mathcal{A}(X),(m, k)=1$, and $\mu$ is a set function satisfying

$$
\mu\left(A_{i+1}\right)+\mu\left(A_{i+2}\right)+\cdots+\mu\left(A_{i+m}\right)=1 \quad \text { for } i=1, \ldots, k
$$

(indices $\bmod k$ ), then $\mu\left(A_{i}\right)=1 / m$ for all $i=1, \ldots, k$.

The proof of this lemma for $m$-chains first appeared in [5], where $m$ chains were first used. Here we give for completeness a short elegant proof shared with the author by J. Aarnes.

Proof. Let $x_{i}=\mu\left(A_{i}\right)$. From (1) we get $\sum_{j=1}^{m} x_{i+j}=1$ and also $\sum_{j=0}^{m-1} x_{i+j}=1$. Subtraction yields $x_{i}=x_{i+m}$, and hence $x_{i}=x_{i+p m}$, $p=0,1, \ldots$ In particular $x_{1}=x_{1+p m}$ for all $p$. Since $(m, k)=1, m$ is a generator for the cyclic group $\mathbb{Z}_{k}$. Hence $x_{1}=\cdots=x_{k}$, and then (1) implies that $x_{i}=1 / m$ for all $i=1, \ldots, k$. 
Definition 2.17. A finite set $F$ is solidifiable in $A$ if there exists $C \in$ $\mathcal{C}_{\mathrm{s}}(X)$ such that $F \cap A \subseteq C \subseteq A$. A finite set is called solidifiable if it is solidifiable in every open connected subset of $X$.

Example 2.18. Any finite set in $\mathbb{R}^{n}, S^{n}$ or the interior of $B^{n}$ for $n \geq 2$ and any finite set in $B^{n}$ for $n \geq 3$ is solidifiable. A finite set in $B^{2}$ that includes boundary points may not be solidifiable. For example, if $X$ is the unit square, $x$ and $y$ are two opposite corner points, and $F$ is a finite set containing points on opposite sides of $X$, then for the open connected set $U=X \backslash\{x, y\}$ there is no closed solid set $C$ with $F \subseteq C \subseteq U$.

Definition 2.19. An $m$-chain $\mathcal{C}=\left\{C_{1}, \ldots, C_{2 m+1}\right\}$ is a solid extension of an $m$-chain $\mathcal{J}=\left\{J_{1}, \ldots, J_{2 m+1}\right\}$ of finite sets if $C_{i} \in \mathcal{C}_{\mathrm{s}}(X)$ and $C_{i} \cap$ $\bigcup \mathcal{J}=J_{i}$ for $i=1, \ldots, 2 m+1$.

EXAMPLE 2.20. Suppose that $X$ is a space for which any closed arc is a closed solid set and for any finite ordered subset of $X$ there exists a simple closed curve that contains this finite set and preserves the order of its points. Such spaces include $S^{n}, \mathbb{R}^{n}$, and the interior of $B^{n}$ for $n \geq 2$. Suppose that $\left\{J_{1}, \ldots, J_{2 m+1}\right\}$ is a basic $m$-chain of finite sets in $X$, i.e. $J_{i}=S_{2 i-1} \sqcup S_{2 i}$ for some disjoint family $\left\{S_{1}, \ldots, S_{2 m+1}\right\}$ of finite sets. Suppose that a simple closed curve in $X$ goes through the points of $S_{1}, \ldots, S_{2 m+1}$ in that order. For $i=1, \ldots, 2 m+1$ let $C_{i}$ be the part of the curve which is an arc beginning at the first point of $S_{2 i-1}$ and ending at the last point of $S_{2 i}$ (indices are mod $2 m+1)$. Then $\left\{C_{1}, \ldots, C_{2 m+1}\right\}$ is a solid extension of the basic $m$-chain $\left\{J_{1}, \ldots, J_{2 m+1}\right\}$.

Proposition 2.21. Suppose $X$ is a space for which there exists $m \geq 1$ such that any basic $m$-chain of finite sets has a solid extension. Then $X$ has no cut points.

Proof. If $\{x\}$ is a cut point and $X \backslash\{x\}=V \sqcup W$ then any solid set that contains points from both $V$ and $W$ must also contain $x$. It is easy to give an example of a basic $m$-chain of finite sets that does not have a solid extension.

Lemma 2.22. Let $X$ be a q-space with no cut points. Suppose $E$ is a finite set, $\left\{F_{1}, \ldots, F_{k}\right\}$ is a disjoint family of subsets of $E$, and $A$ is any open solid set containing $\bigsqcup_{i=1}^{k} F_{i}$. Assume $E$ is solidifiable. Then there exists a disjoint family $\left\{C_{1}, \ldots, C_{k}\right\}$ of closed solid sets such that $C_{i} \subseteq A$ and $C_{i} \cap E=F_{i}$. Any disjoint family $\left\{C_{1}, \ldots, C_{n}\right\}$ of closed solid sets, $1 \leq n<k$, such that $C_{i} \subseteq A$ and $C_{i} \cap E=F_{i}$ for $i=1, \ldots, n$ extends to a disjoint family $\left\{C_{1}, \ldots, C_{k}\right\}$ of closed solid sets with $C_{i} \subseteq A$ and $C_{i} \cap E=F_{i}$ for $i=1, \ldots, k$. 
Proof. We construct the desired sets $C_{i}$ by induction. Let $\widetilde{F}_{i}=E \backslash F_{i}$ for each $i, C_{0}=\emptyset$, and $A^{\mathrm{c}}=X \backslash A$. For each $i$ the set $A \backslash \bigsqcup_{j=0}^{i-1} C_{j}$ is open connected by Proposition 2.5. Then $\left(A \backslash \bigsqcup_{j=0}^{i-1} C_{j}\right) \backslash \widetilde{F}_{i}$ is also open connected by Proposition 2.5 and contains $F_{i}$, so there exists $C_{i} \in \mathcal{C}_{\mathrm{s}}(X)$ such that $F_{i} \subseteq C_{i} \subseteq\left(A \backslash \bigsqcup_{j=0}^{i-1} C_{j}\right) \backslash \widetilde{F}_{i}$. Notice that $C_{i} \cap E=F_{i}$.

In the previous lemma the condition that $X$ has no cut points can be weakened. We use only the fact that none of the points of $E$ is a cut point.

REMARK 2.23. If $X$ has a cut point then the extreme topological measures are not dense in the set of all topological measures on $X$. If $x$ is a cut point of $X$ then by [13] each extreme topological measure is supported on the union of $\{x\}$ with one of the components of $X \backslash\{x\}$. Any topological measure whose support contains more than one component of $X \backslash\{x\}$ cannot be approximated by extreme topological measures.

3. Main theorem. The proof of the main theorem is based on a series of lemmas.

Lemma 3.1. Let $\mathcal{W}$ be a finite family of sets and $b: \mathcal{W} \rightarrow[0,1]$ be a function such that $\sum_{U \in \mathcal{D}} b(U)<1$ for every disjoint collection $\mathcal{D} \in \mathrm{DC}(\mathcal{W})$. Then there exist $m \in \mathbb{N}$ and a function $n: \mathcal{W} \rightarrow \mathbb{N}$ such that

(i) $2[n(U) / 2] \geq(2 m+1) b(U)$ for each $U \in \mathcal{W}$,

(ii) $\sum_{U \in \mathcal{D}} n(U) \leq 2 m+1$ for every $\mathcal{D} \in \mathrm{DC}(\mathcal{W})$,

(iii) for each $U \in \mathcal{W}$ there exists $\mathcal{D} \in \operatorname{MDC}(\mathcal{W})$ with $\sum_{V \in \mathcal{D}} n(V)=$ $2 m+1$ and $U \in \mathcal{D}$.

Proof. Since we get a basis for the topology on $T M(X)$ by considering functions $b$ with values in a dense subset of $[0,1]$, we may without loss of generality assume that there are integers $m(U)$ and an integer $m$ such that for all $U \in \mathcal{W}$,

$$
b(U)=\frac{2 m(U)}{2 m+1} .
$$

One way to prove the lemma is by providing an algorithm for obtaining the function $n$. Set initially $n(U)=2 m(U)$ for each $U \in \mathcal{W}$ and $\mathcal{W}^{\prime}=\mathcal{W}$.

Let $U \in \mathcal{W}^{\prime} \subseteq \mathcal{W}$. Then $U$ belongs to a maximal disjoint collection $\mathcal{C} \in \operatorname{MDC}(\mathcal{W})$. Let $\mathcal{D} \in \operatorname{MDC}(\mathcal{W})$ be the maximal disjoint collection which minimizes the quantity

$$
2 m+1-\sum_{V \in \mathcal{C}, V \neq U} n(V)
$$

over all maximal disjoint collections $\mathcal{C}$ containing $U$. Let

$$
n(U)=2 m+1-\sum_{V \in \mathcal{D}, V \neq U} n(V)
$$


It is clear that $\sum_{U \in \mathcal{D}} n(U)=2 m+1$ and that for each $U \in \mathcal{W}$ we have $n(U) \geq 2 m(U)=(2 m+1) b(U)$, i.e. $2[n(U) / 2] \geq(2 m+1) b(U)$.

Now let $\mathcal{W}^{\prime} \backslash \bigcup\left\{\mathcal{C}: \mathcal{C} \in \operatorname{MDC}(\mathcal{W})\right.$ with $\left.\sum_{U \in \mathcal{C}} n(U)=2 m+1\right\}$ (where one such maximal disjoint collection is $\mathcal{D}$ ) be the new $\mathcal{W}^{\prime}$. Repeat the algorithm for the new $\mathcal{W}^{\prime}$. When $\mathcal{W}^{\prime}=\emptyset$ the desired function $n$ is produced. Notice that for any $V \in \mathcal{W}$ such that $\{V\} \in \operatorname{MDC}(\mathcal{W})$ (these are sets $V$ with the property that $V$ intersects every set in $\mathcal{W}$ ) we have $n(V)=$ $2 m+1$.

An alternative proof of the lemma runs as follows. The set $\mathcal{N}$ of all functions $n$ satisfying the first two conditions of the lemma is not empty (for example, it includes $n$ defined by $n(U)=2 m(U)$ ). The reasoning in the above algorithm shows that the last condition of the lemma is satisfied if we chose $n \in \mathcal{N}$ to be maximal.

Lemma 3.2. Let $\mathcal{W}$ be a finite family of open subsets of $X$ and $n: \mathcal{W} \rightarrow$ $\mathbb{N}$ be a function. Then there is a function $R: \mathcal{W} \rightarrow \mathcal{P}(\mathcal{P}(X))$ such that:

(a) the members of $R(U)$ are nonempty finite disjoint subsets of $U$,

(b) $|R(U)|=n(U)$ for each $U \in \mathcal{W}$,

(c) if $U \neq V$ and $U \cap V \neq \emptyset$ then $S \cap T \neq \emptyset$ for any pair of sets $S \in R(U)$, $T \in R(V)$

(d) the cardinality of the set $E=\bigcup_{U \in \mathcal{W}}(\bigcup R(U))$ is odd,

(e) if $R$ is a function satisfying (a), (b) and (c), $S \in R(U), T \in R(V)$, and $n(V) \geq 2$ then $S \subseteq T$ implies $U=V$ and $S=T$.

Proof. Suppose first that $\mathcal{W}=\{U\}$. Let $l$ be an odd number exceeding $n(U)$. Choose $l$ different points in $U$ and form $n(U)$ finite disjoint sets, which will constitute $R(U)$. Then (a)-(d) are satisfied. It is easy to see how (d) can be satisfied in general, and we will prove (a)-(c) by induction on $|\mathcal{W}|$. Assume that (a)-(c) hold for any $\mathcal{W}$ with $|\mathcal{W}| \leq k$. Let $|\mathcal{W}|=k+1$, and let $U \in \mathcal{W}$. Then (a)-(c) hold for $\mathcal{W}^{\prime}=\mathcal{W} \backslash\{U\}$. Using the argument at the beginning of the proof we can choose $R(U)$ so that (a) and (b) hold for $\mathcal{W}$. Now suppose that $V \in \mathcal{W}^{\prime}, U \cap V \neq \emptyset$. Let $R(U)=\left\{S_{1}, \ldots, S_{n(U)}\right\}$ and $R(V)=\left\{T_{1}, \ldots, T_{n(V)}\right\}$. In the nonempty open set $U \cap V$ pick $n(U) n(V)$ different points $p_{i j}, i=1, \ldots, n(U), j=1, \ldots, n(V)$. Modify each set $S_{i}$ by adding points $p_{i j}, j=1, \ldots, n(V)$, and each set $T_{j}$ by adding points $p_{i j}, i=1, \ldots, n(U)$. The result of this modification is that while all sets $S_{i}$ are disjoint, and all sets $T_{j}$ are disjoint, we have $p_{i j} \in S_{i} \cap T_{j} \neq \emptyset$ for every $i=1, \ldots, n(U)$ and every $j=1, \ldots, n(V)$. Hence, (c) is satisfied for $\mathcal{W}$. To prove (e), suppose that $n(V) \geq 2$ and take disjoint sets $T, T^{\prime} \in R(V)$. If $U \neq V$ then $S \subseteq T$ implies $U \cap V \neq \emptyset$ and then by (c) we also have $S \cap T^{\prime} \neq \emptyset$, which makes $S \subseteq T$ impossible. Therefore, we must have $U=V$ and $S=T$. 
Lemma 3.3. Let the function $R: \mathcal{W} \rightarrow \mathcal{P}(\mathcal{P}(X))$ and the set $E$ be as in the previous lemma. Consider families (of subsets of $E$ ) $\mathcal{E}=\{S: S \in$ $R(U), U \in \mathcal{W}\}$ and $\mathcal{F}_{m}=\{S \sqcup T: S \in R(U), T \in R(V), U, V \in \mathcal{D}, \mathcal{D} \in$ $\operatorname{MDC}(\mathcal{W})$ with $\left.\sum_{U \in \mathcal{D}} n(U)=2 m+1\right\}$ (note that $U=V$ is allowed). Let $\mathcal{F}=\mathcal{F}_{m} \cup\{E\}$. Then:

(a) the family $\mathcal{E}$ has order $2 m+1$,

(b) each set in $\mathcal{F}_{m}$ belongs to a basic m-chain,

(c) the families $\mathcal{F}, \mathcal{F}_{m}$ both have order $m$ and contain an $m$-chain.

Proof. (a) Suppose $\mathcal{S}$ is a disjoint subfamily of $\mathcal{E}$. Let $\mathcal{D}=\{U: S \in$ $R(U), S \in \mathcal{S}\}$. From part (c) in Lemma 3.2 we see that $\mathcal{D} \in \mathrm{DC}(\mathcal{W})$. Then by (ii) in Lemma 3.1 ,

$$
|\mathcal{S}| \leq \sum_{U \in \mathcal{D}} n(U) \leq 2 m+1 .
$$

If $\mathcal{C} \in \operatorname{MDC}(\mathcal{W})$ is any maximal disjoint collection with $\sum_{U \in \mathcal{C}} n(U)=2 m+1$ then by Lemmas 3.1 and $3.2, \mathcal{S}^{\prime}=\{S: S \in R(U), U \in \mathcal{C}\}$ is a disjoint subfamily of $\mathcal{E}$ with exactly $2 m+1$ sets. Therefore, the order of $\mathcal{E}$ is $2 m+1$.

(b) Let $S \sqcup T$ be an element of $\mathcal{F}_{m}$. Here $S \in R(U), T \in R(V), U, V \in \mathcal{C}$, where $\mathcal{C}$ is a maximal disjoint collection with $\sum_{U \in \mathcal{C}} n(U)=2 m+1$. Let $\mathcal{S}^{\prime}$ be as above. Example 2.14 applied to $\mathcal{S}^{\prime}$ shows that $S \sqcup T$ belongs to a basic $m$-chain.

(c) Clearly, both $\mathcal{F}$ and $\mathcal{F}_{m}$ contain an $m$-chain. If the family $\mathcal{F}_{m}$ were to contain $m+1$ disjoint sets, that would imply that $\mathcal{E}$ has $2 m+2$ disjoint sets, which contradicts (a). Thus, the order of $\mathcal{F}_{m}$ is $m$, and the same is true for $\mathcal{F}$, since the set $E$ intersects every set in $\mathcal{F}_{m}$.

Lemma 3.4. Let $\mathcal{W}$ be a finite family of open subsets of $X$, and let the set $E$ and families $\mathcal{F}$ and $\mathcal{F}_{m}$ be as in Lemma 3.3. Let $\alpha: \mathcal{F} \rightarrow[0,1]$ be the function which takes the value $1 / m$ on $\mathcal{F}_{m}$ and the value 1 on $E$. Then $\alpha$ is a pre-supermeasure. The corresponding supermeasure $\nu$ (given by Theorem 2.11) is such that $\nu(J)=1 / m$ for every $J \in \mathcal{F}_{m}$.

Proof. First, recall that the set $E$ intersects every set in the family $\mathcal{F}$. To show that $\alpha$ is a pre-supermeasure, we need to check condition $(*)$ from Definition 2.10 with $\mathcal{H}$ being a disjoint subfamily of $\mathcal{F}$. If $B=E$ then $(*)$ holds because the order of $\mathcal{F}$ is $m$. Let $B \in \mathcal{F}_{m}$, i.e. $B=S \sqcup T$, where $S \in R(U), T \in R(V), U, V \in \mathcal{C}$ and $\mathcal{C}$ is a maximal disjoint collection with $\sum_{U \in \mathcal{C}} n(U)=2 m+1$. As in the proof of Lemma 3.3, using the maximal disjoint collection $\mathcal{C}$ we get a disjoint subfamily of $\mathcal{E}$ with exactly $2 m+1$ sets. This subfamily of $\mathcal{E}$ contains $2 m-1$ disjoint sets other than $S, T$. Notice that none of the sets in $\mathcal{H}$ is $E$. The assumption that $|\mathcal{H}| \geq 2$ would imply that there were at least $2 m+3$ disjoint sets in $\mathcal{E}$, which is impossible by Lemma 3.3. Therefore, $|\mathcal{H}|=1$, and $(*)$ is satisfied. 
If $J_{1}, \ldots, J_{m}$ are disjoint sets in $\mathcal{F}_{m}$ then, by Theorem 2.11,

$$
1=\sum_{i=1}^{m} \alpha\left(J_{i}\right) \leq \sum_{i=1}^{m} \nu\left(J_{i}\right) \leq \nu\left(J_{1} \sqcup \cdots \sqcup J_{m}\right) \leq 1
$$

and so $\nu\left(J_{i}\right)=1 / m$, since $\nu\left(J_{i}\right) \geq \alpha\left(J_{i}\right)=1 / m$ for each $i$. Hence, $\nu(J)=$ $1 / m$ for every $J \in \mathcal{F}_{m}$ because by Lemma 3.3 each set $J \in \mathcal{F}_{m}$ belongs to an $m$-chain.

Lemma 3.5. Let the supermeasure $\nu$ and the finite set $E$ be as in Lem$m a 3.4$, and $i: E \rightarrow X$ be the inclusion. Suppose $E$ is solidifiable and $X$ is a q-space with no cut points in which every basic $m$-chain of subsets of $E$ has a solid extension. Then the topological measure $\mu=i_{*} \nu$ (given by Proposition 2.9) is extreme.

Proof. Let $\mu=i_{*} \nu$ be given by Proposition 2.9. Assume $\mu=\frac{1}{2} \mu_{1}+\frac{1}{2} \mu_{2}$, i.e.

$$
\mu(A)=\frac{1}{2} \mu_{1}(A)+\frac{1}{2} \mu_{2}(A)
$$

for all $A \in \mathcal{A}(X)$. We need to show that $\mu=\mu_{1}=\mu_{2}$, i.e. $\mu(A)=\mu_{1}(A)=$ $\mu_{2}(A)$ for all $A \in \mathcal{A}(X)$. It is enough to check this for $A \in \mathcal{A}_{\mathrm{s}}(X)$. Notice that for any open or closed set $A$ with $\mu(A)=1$ or 0 from (2) it follows that also $\mu_{1}(A)=1$ or 0 .

First we show that for $K \in \mathcal{C}_{\mathrm{s}}(X)$ with $K \cap E=J$ (for any set $J \in \mathcal{F}_{m}$ ),

$$
\mu(K)=\mu_{1}(K)=\mu_{2}(K)=1 / m=\nu(J) .
$$

Let $C_{1}=K$. By Lemma $3.3, J$ is an element of a basic $m$-chain $\mathcal{J}=$ $\left\{J_{1}, \ldots, J_{2 m+1}\right\}$ in $\mathcal{F}_{m}$, which has a solid extension, a chain $\left\{C_{1}, \ldots, C_{2 m+1}\right\}$ of closed solid sets in $X$ such that $C_{i} \cap \bigcup \mathcal{J}=J_{i}$ for $i=1, \ldots, 2 m+1$. Here $\bigcup \mathcal{J} \subseteq E$. By Lemma 3.4, $\mu\left(C_{i}\right)=\nu\left(C_{i} \cap E\right) \geq \nu\left(J_{i}\right)=1 / m$ for all $i=$ $1, \ldots, 2 m+1$. The sets $C_{i+1}, \ldots, C_{i+m}$ are disjoint for all $i=1, \ldots, 2 m+1$ (indices are $\bmod 2 m+1$ ), so we have

$$
\mu\left(C_{i+1} \sqcup \cdots \sqcup C_{i+m}\right)=\mu\left(C_{i+1}\right)+\cdots+\mu\left(C_{i+m}\right)=1 .
$$

The closed set $C=C_{i+1} \sqcup \cdots \sqcup C_{i+m}$ has $\mu(C)=1$ so we also have $\mu_{1}(C)=1$, i.e.

$$
\mu_{1}\left(C_{i+1} \sqcup \cdots \sqcup C_{i+m}\right)=\mu_{1}\left(C_{i+1}\right)+\cdots+\mu_{1}\left(C_{i+m}\right)=1
$$

for all $i=1, \ldots, 2 m+1$ (indices are $\bmod 2 m+1$ ). Then Lemma 2.16 tells us that $\mu_{1}\left(C_{i}\right)=1 / m$ for all $i=1, \ldots, 2 m+1$. Therefore, $\mu_{1}\left(C_{i}\right)=$ $1 / m=\mu\left(C_{i}\right)$ for all $i=1, \ldots, 2 m+1$. Since $\mu=\frac{1}{2} \mu_{1}+\frac{1}{2} \mu_{2}$ we have $\mu_{1}\left(C_{i}\right)=\mu_{2}\left(C_{i}\right)=\mu\left(C_{i}\right)=1 / m$ for all $i=1, \ldots, 2 m+1$. In particular, $\mu(K)=\mu_{1}(K)=\mu_{2}(K)=1 / m=\nu(J)$.

Let $A$ be a solid set and let $J^{\prime}=A \cap E$, so $\mu(A)=\nu\left(J^{\prime}\right)$. Suppose first that $\left|J^{\prime}\right|<|E| / 2$. By the construction of $\nu$ in [4], we may assume that 
$\nu\left(J^{\prime}\right)=\sum_{J \in \mathcal{H}} \alpha(J)$ for some disjoint subfamily $\mathcal{H}$ of $\mathcal{F}$ with $\bigsqcup \mathcal{H} \subseteq J^{\prime}$. By the regularity of topological measures we may assume that $A$ is open solid, and then by Lemma 2.22 we may find disjoint closed solid sets $K_{J} \subseteq A$, $J \in \mathcal{H}$ such that $J=K_{J} \cap E$. Then using (3) we have

$$
\mu(A)=\nu\left(J^{\prime}\right)=\sum_{J \in \mathcal{H}} \alpha(J)=\sum_{J \in \mathcal{H}} \mu\left(K_{J}\right)=\sum_{J \in \mathcal{H}} \mu_{1}\left(K_{J}\right) \leq \mu_{1}(A) .
$$

Similarly, $\mu(A) \leq \mu_{2}(A)$. Then $\mu(A)=\mu_{1}(A)=\mu_{2}(A)$. We see that the same equality holds for $A \in \mathcal{A}_{\mathrm{S}}(X)$ with $|A \cap E|>|E| / 2$ by taking the complement of $A$. Hence, $\mu$ is extreme.

REMARK 3.6. The technique for showing that $\mu$ is extreme is a reworking of a technique that first appeared in [5].

TheOREm 3.7. Let $X$ be a q-space in which any finite set is solidifiable and any basic $m$-chain of finite sets has a solid extension. Then extreme topological measures are dense in the set of all topological measures on $X$.

Proof. Let $\widehat{U}(\mathcal{W}, b)$ be a nonempty basic open set. Here $\mathcal{W}$ is a nonempty finite collection of open solid subsets of $X$ and $b: \mathcal{W} \rightarrow[0,1]$ is a function. Our goal is to find an extreme topological measure $\mu$ in $\widehat{U}(\mathcal{W}, b)$. We may assume that none of the sets $U \in \mathcal{W}$ is empty. We may also assume that $X \in \mathcal{W}$. (See Lemma 3.2 in [4].) Since our basic open set is nonempty, it follows that $\sum_{U \in \mathcal{D}} b(U)<1$ for every disjoint collection $\mathcal{D} \in \mathrm{DC}(\mathcal{W})$, and in particular, each $b(U)<1$. Indeed, consider a topological measure $\lambda \in \widehat{U}(\mathcal{W}, b)$. If $\sum_{U \in \mathcal{D}} b(U) \geq 1$ for a disjoint collection $\mathcal{D} \in \operatorname{DC}(\mathcal{W})$ then we would have

$$
\lambda(X) \geq \sum_{U \in \mathcal{D}} \lambda(U)>\sum_{U \in \mathcal{D}} b(U) \geq 1
$$

which contradicts the normalization of $\lambda$.

Now we may apply Lemma 3.1 to get a function $n: \mathcal{W} \rightarrow \mathbb{N}$. Apply Lemma 3.2 for $\mathcal{W}$ and $n$. Let families $\mathcal{E}$ and $\mathcal{F}_{m}$ be as in Lemma 3.3 and the set $E$ be as in Lemma 3.2. By Proposition 2.21, $X$ has no cut points, so we apply Lemma 3.5 to obtain an extreme topological measure $\mu$. It only remains to show that $\mu$ is in the basic open set $\widehat{U}(\mathcal{W}, b)$. Let $U \in \mathcal{W}$ be in a maximal disjoint collection $\mathcal{D}$. By Lemma 3.2, the set $U$ contains $n(U)$ disjoint members of $R(U)$, so $U \cap E$ contains $[n(U) / 2]$ disjoint sets from $\mathcal{F}_{m}$, each of which by Lemma 3.4 has $\nu$ measure $1 / \mathrm{m}$. According to Proposition 2.9, $\mu(U)=\nu(U \cap E)$. Using (i) from Lemma 3.1 we get

$$
\mu(U)=\nu(U \cap E) \geq\left[\frac{n(U)}{2}\right] \frac{1}{m}>\left[\frac{n(U)}{2}\right] \frac{2}{2 m+1} \geq b(U) .
$$

REMARK 3.8. Suppose that a nonempty basic open set $\widehat{U}(\mathcal{W}, b)$ is such that $\operatorname{MDC}(\mathcal{W})=\{\{U\}: U \in \mathcal{W}\}$, i.e. the family $\mathcal{W}$ has the pairwise 
nonempty intersection property: $U \cap V \neq \emptyset$ for any $U, V \in \mathcal{W}$. In this case there is a simple topological measure in $\widehat{U}(\mathcal{W}, b)$ (see [12]).

Theorem 3.7 can be improved, as D. Grubb pointed out to the author.

TheOrem 3.9. Let $X$ be a q-space and $Y$ be a dense open subset of $X$ such that any finite subset of $Y$ is solidifiable and any basic $m$-chain of finite subsets of $Y$ has a solid extension. Then extreme topological measures are dense in the set of all topological measures on $X$.

This theorem holds because one may choose the sets $R(U)$ in Lemma 3.2 as subsets of $Y$.

ExAMPLE 3.10. Theorems 3.7 and 3.9 and Examples 2.18 and 2.20 show that among the spaces on which extreme topological measures are dense in the collection of all topological measures are the spheres $S^{n}$, balls $B^{n}$, and projective planes $\mathbb{R} P^{n}$ for $n \geq 2$.

The extreme topological measure $\mu$ constructed in Theorem 3.7 is finitely defined. Hence, we get the following corollary, which implies Theorem 3.4 in [4] under the assumptions of Theorem 3.9.

Corollary 3.11. Let $X$ be a q-space and $Y$ be a dense open subset of $X$ such that any finite subset of $Y$ is solidifiable and any basic $m$-chain of finite subsets of $Y$ has a solid extension. Then extreme finitely defined topological measures are dense in the set of all topological measures on $X$.

Note that the families of finitely defined topological measures and extreme topological measures intersect without one containing the other. The topological measure in Example 2.3 is extreme but not finitely defined, and from Proposition 3.16 in [6] it is easy to get finitely defined topological measures that are not extreme.

Acknowledgments. The author would like to thank the anonymous referees for excellent comments that greatly improved the paper.

\section{References}

[1] J. F. Aarnes, Quasi-states on $C^{*}$-algebras, Trans. Amer. Math. Soc. 149 (1970), 601-625.

[2] —, Quasi-states and quasi-measures, Adv. Math. 86 (1991), 41-67.

[3] - Construction of non-subadditive measures and discretization of Borel measures, Fund. Math. 147 (1995), 213-237.

[4] J. F. Aarnes and S. V. Butler, Super-measures and finitely defined topological measures, Acta Math. Hungar. 99 (2003), 33-42.

[5] S. V. Butler, Q-functions, extreme points and density theorems in the space of quasimeasures, Ann Arbor, MI, UMI Dissertation Services, 2000.

[6] - Density in the space of topological measures, Fund. Math. 174 (2002), 239-251. 
[7] S. V. Butler, Q-functions and extreme topological measures, J. Math. Anal. Appl. 307 (2005), 465-479.

[8] D. J. Grubb, Irreducible partitions and the construction of quasi-measures, Trans. Amer. Math. Soc. 353 (2001), 2059-2072.

[9] - - private communication.

[10] D. J. Grubb and T. Laberge, Additivity of quasi-measures, Proc. Amer. Math. Soc. 126 (1998), 3007-3012.

[11] —, - Spaces of quasi-measures, Canad. Math. Bull. 42 (1999), 291-297.

[12] F. F. Knudsen, Topology and the construction of extreme quasi-measures, Adv. Math. 120 (1996), 302-321.

[13] T. Laberge, Supports of quasi-measures, Houston J. Math. 24 (1998), 301-312.

Department of Mathematics

University of Illinois at Urbana-Champaign

273 Altgeld Hall, 1409 West Green Street

Urbana, IL 61801, U.S.A.

E-mail: svbutler@uiuc.edu

Received 12 September 2005;

in revised form 22 June 2006 